\title{
Altered pulmonary blood volume distribution as a biomarker for predicting outcomes in COVID-19 disease
}

\author{
Michael F. Morris $\mathbb{1}^{1,2}$, Yash Pershad ${ }^{3}$, Paul Kang ${ }^{4}$, Lauren Ridenour ${ }^{2}$, Ben Lavon ${ }^{5}$, Maarten Lanclus ${ }^{5}$, \\ Rik Godon ${ }^{5}$, Jan De Backer ${ }^{5}$ and Marilyn K. Glassberg $\mathbb{1}^{2,6}$
}

${ }^{1}$ Dept of Radiology, Banner - University Medical Center Phoenix, Phoenix, AZ, USA. ${ }^{2}$ Dept of Medicine, Banner - University Medical Center Phoenix, Phoenix, AZ, USA. ${ }^{3}$ Dept of Bioengineering, Stanford University, Palo Alto, CA, USA. ${ }^{4}$ Dept of Biostatistics, University of Arizona College of Public Health, Phoenix, AZ, USA. ${ }^{5}$ FLUIDDA, New York City, NY, USA. ${ }^{6}$ Division of Pulmonary Medicine, Critical Care, and Sleep Medicine, University of Arizona College of Medicine - Phoenix, Phoenix, AZ, USA.

Corresponding author: Michael F. Morris (michael.morris@bannerhealth.com)

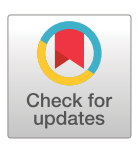

This version is distributed under the terms of the Creative Commons Attribution Non-Commercial Licence 4.0. For commercial reproduction rights and permissions contact permissions@ersnet.org

This article has supplementary material available from erj.ersjournals.com

This article has an editorial commentary: https://doi.org/10. 1183/13993003.00811-2021

Received: 9 Nov 2020 Accepted: 3 Feb 2021

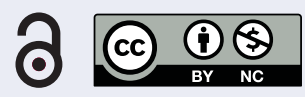

Shareable abstract (@ERSpublications)

BV5\% derived from chest CT may serve as an imaging biomarker for predicting adverse outcomes in patients with COVID-19 seeking acute medical care https://bit.ly/2Zg18Vv

Cite this article as: Morris MF, Pershad Y, Kang P, et al. Altered pulmonary blood volume distribution as a biomarker for predicting outcomes in COVID-19 disease. Eur Respir J 2021; 58: 2004133 [DOI: 10.1183/13993003.04133-2020].

\section{Abstract}

Introduction Evidence suggests that vascular inflammation and thrombosis may be important drivers of poor clinical outcomes in patients with COVID-19. We hypothesised that a significant decrease in the percentage of blood volume in vessels with a cross-sectional area between 1.25 and $5 \mathrm{~mm}^{2}$ relative to the total pulmonary blood volume (BV5\%) on chest computed tomography (CT) in COVID-19 patients is predictive of adverse clinical outcomes.

Methods We performed a retrospective analysis of chest CT scans from 10 hospitals across two US states in 313 COVID-19-positive and 195 COVID-19-negative patients seeking acute medical care.

Results BV5\% was predictive of outcomes in COVID-19 patients in a multivariate model, with a BV5\% threshold below 25\% associated with OR 5.58 for mortality, OR 3.20 for intubation and OR 2.54 for the composite of mortality or intubation. A model using age and BV5\% had an area under the receiver operating characteristic curve of 0.85 to predict the composite of mortality or intubation in COVID-19 patients. BV5\% was not predictive of clinical outcomes in patients without COVID-19.

Conclusions The data suggest BV5\% as a novel biomarker for predicting adverse outcomes in patients with COVID-19 seeking acute medical care.

\section{Introduction}

The severe acute respiratory syndrome coronavirus 2 (SARS-CoV-2) pandemic has placed substantial pressure on many aspects of the healthcare system [1] and patients presenting to the hospital with COVID-19 disease have significant risk for progression to respiratory failure or death [2, 3]. Increasing evidence suggests that vascular inflammation and thrombosis may be important drivers of poor clinical outcomes in patients with COVID-19 [4]. Autopsy studies demonstrate that pulmonary endothelialitis and microangiopathy are significantly more common in COVID-19 compared with other viral respiratory illnesses [5].

Changes in the pulmonary vasculature on chest computed tomography (CT) may reflect the angiocentric injury caused by COVID-19 [6]. Quantitative analysis of chest CT scans from patients with COVID-19 demonstrates a significant decrease in the percentage of blood vessels with a cross-sectional area between 1.25 and $5 \mathrm{~mm}^{2}$ (BV5\%; see Methods) [7]. Given the effects of COVID-19 on pulmonary microcirculation, we hypothesised that BV5\% reduction on chest CT in COVID-19 patients is associated with adverse outcomes of respiratory failure requiring intubation or death. 


\section{Methods}

This was an institutional review board-approved retrospective study of patients seeking acute medical care within a large integrated healthcare network in the USA from 1 March 2020 to 30 June 2020. Patients presenting to the emergency department or directly admitted to the hospital and undergoing a chest CT within $24 \mathrm{~h}$ of presentation and COVID-19 testing were eligible for participation (figure 1). After identification of the COVID-19-positive cohort, a randomly selected group of COVID-19-negative patients was chosen in order to achieve a target study ratio of 60\% COVID-19-positive and 40\% COVID-19-negative cases for analysis.

Patient demographic information, treatment course and outcome data were obtained from the clinical information system through a combination of custom-coded data retrieval and manual abstraction. All COVID-19 testing was performed using nasopharyngeal swabs, with PCR assays processed in a central laboratory. In patients with a negative COVID-19 PCR test, the medical record was checked for results of an additional COVID-19 test within 14 days. Initial blood laboratory values obtained within $24 \mathrm{~h}$ of the CT scan were recorded.

\section{Semiquantitative CT analysis}

A fellowship-trained cardiothoracic radiologist visually scored CT scans for the severity of lung opacification, blinded to the patient's COVID-19 status and clinical outcome. A semiquantitative scoring method correlating with disease severity and short-term outcomes in COVID-19 patients was utilised [8, 9]. Each lobe was scored as: 0 (no opacification), 1 (<5\% opacification), 2 (5-25\% opacification), 3 (26-50\% opacification), 4 (51-75\% opacification) or 5 (>75\% opacification). The severity score for each CT was calculated as the sum of the five lobar scores (range 0-25).

\section{Quantitative CT analysis}

A recent publication provides a detailed description of the quantitative CT analysis technique utilised to calculate BV5\% as well as the percentage of lung opacification [10]. Briefly, CT scans with slice thickness

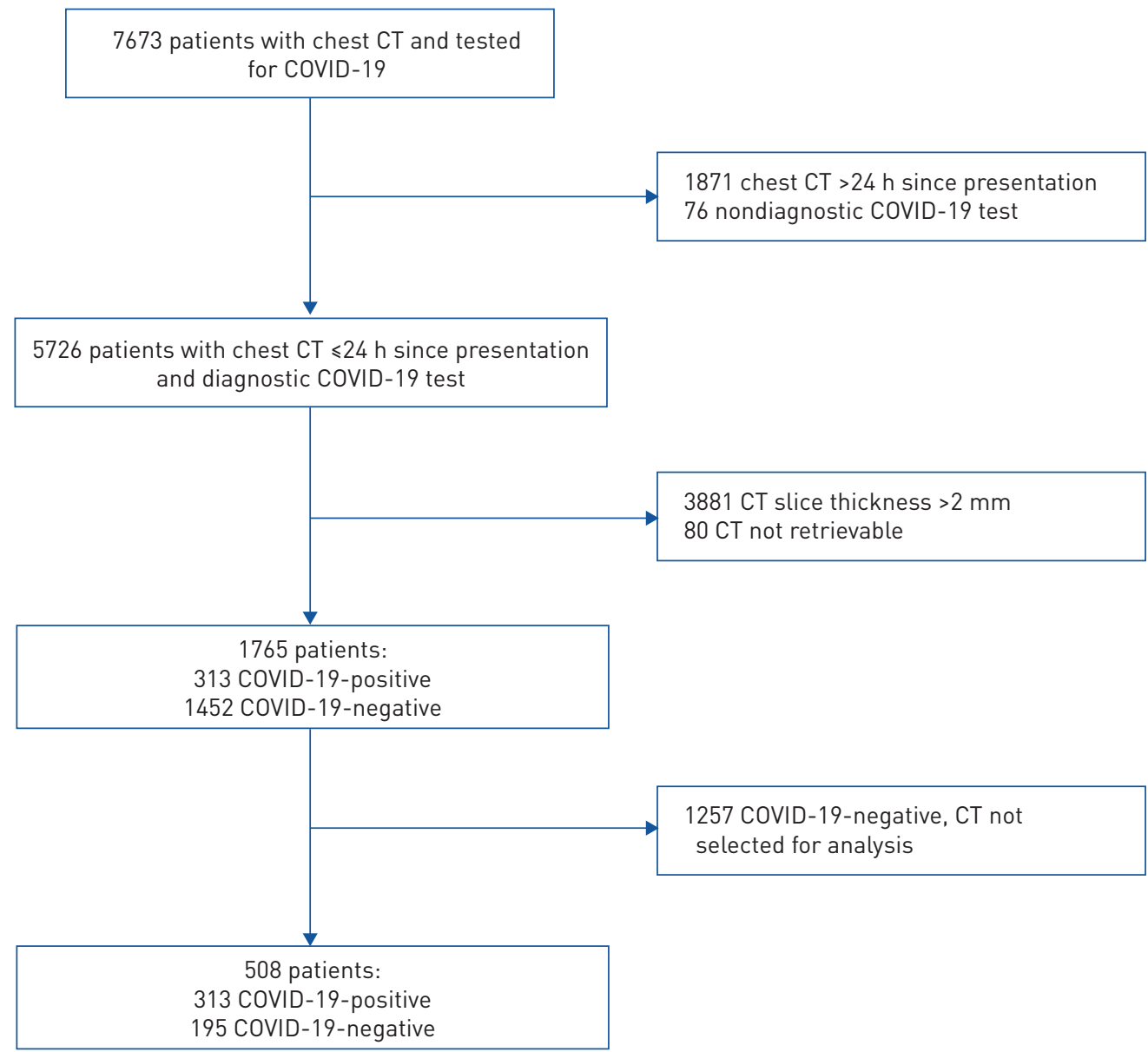

FIGURE 1 Flowchart of eligible patients. CT: computed tomography. 
$\leqslant 2 \mathrm{~mm}$ were used to generate three-dimensional reconstructions of the lung tissue, airways and pulmonary vasculature. An automated algorithm segmented the vasculature by identifying and enhancing cylindrical structures, and excluded airways via Hounsfield unit thresholds. Blood vessels were then clustered into three groups by cross-sectional area. BV5, BV5-10 and BV10 denoted the volume of blood contained in vessels with a cross-sectional area between 1.25 and $5 \mathrm{~mm}^{2}$, between 5 and $10 \mathrm{~mm}^{2}$ and $>10 \mathrm{~mm}^{2}$, respectively. BV5\%, BV5-10\% and BV10\% were calculated as the percentage of blood volume in vessels with a cross-sectional area between 1.25 and $5 \mathrm{~mm}^{2}$, between 5 and $10 \mathrm{~mm}^{2}$ and $>10 \mathrm{~mm}^{2}$, respectively, relative to the total pulmonary blood volume (figure 2).

\section{Statistical analysis}

Patient demographic and clinical characteristics were stratified by COVID-19 testing results, and are reported as mean and standard deviation for continuous variables or frequency and percentage for categorical variables. The Wilcoxon rank sum test was used to assess differences in continuous variables, while Fisher's exact test was used to compare categorical variables. For the outcomes of mortality, intubation and their combination, multivariable logistic regression was implemented to ascertain the odds of the outcomes relative to BV5\% as a continuous and categorical variable. BV5\% was categorised using the Lowess Smoother plot. If the relationship between BV5\% and the log odds of the outcomes was not linear, the inflection point was used to create the threshold for categorisation. Multivariable linear regression was also used to estimate the mean difference in hospital length of stay and the number of intubation days relative to BV5\%. Statistical models were adjusted for covariates that were prognostic for outcomes; thus, age, gender, race, body mass index, tobacco use, diabetes, hyperlipidaemia, hypertension, heart failure, chronic kidney disease, chronic obstructive pulmonary disease (COPD)/emphysema, cerebrovascular disease, cancer, contrast enhanced CT scan, CT findings, anticoagulation treatment, steroid treatment, azithromycin treatment, remdesivir treatment, white blood cell count, lymphocyte count, haematocrit, platelet count and estimated glomerular filtration rate were included in the models. Missing data were handled using multiple imputation with 10 imputations. Two-sided p-values $<0.05$ were considered statistically significant. Data analyses were conducted using Stata version 15 (StataCorp, College Station, TX, USA).

A logistic regression model from scikit-learn [11] was trained on COVID-19-positive patients to predict the risk of mortality, intubation and their combination. To prevent over-fitting, model performance was assessed in two ways: 1) accuracy of predictions in leave-one-out cross-validation, and 2) area under the receiver operating characteristic curve (AUC) and precision-recall curves after splitting data into training (80\%) and testing (20\%) sets [12]. Differences in AUC were compared using a one-sample z-test of proportions. With the coefficients and intercept determined from training, predictions of risk from the logistic regression model were used to simulate patients of varying age and BV5\%.

\section{Results}

A total of 508 patients from 10 hospitals across Arizona and Colorado were included in the analysis, including 313 COVID-19-positive patients and 195 COVID-19-negative patients (table 1). 55\% of patients
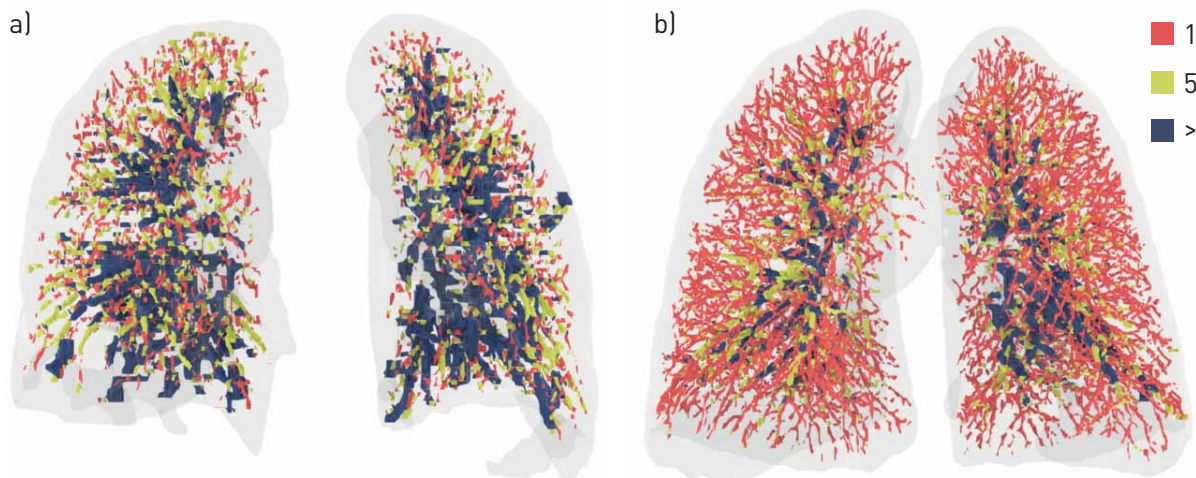

$1.25-5 \mathrm{~mm}^{2}$

$5-10 \mathrm{~mm}^{2}$

$>10 \mathrm{~mm}^{2}$

FIGURE 2 Volume-rendered images of chest computed tomography scans from two different patients with COVID-19, with colour-coded segmentation of the pulmonary vascular cross-sectional area. BV5\%: percentage of blood volume in vessels with a cross-sectional area between 1.25 and $5 \mathrm{~mm}^{2}$ relative to the total pulmonary blood volume. a) Patient with BV5\% of $21 \%$. b) Patient with BV $5 \%$ of $55 \%$. Colour coding denotes blood volume in vessels with a cross-sectional area between 1.25 and $5 \mathrm{~mm}^{2}$ (BV5\%), between 5 and $10 \mathrm{~mm}^{2}$ (BV5-10\%) and $>10 \mathrm{~mm}^{2}$ (BV10\%). 
TABLE 1 Baseline demographic, laboratory and treatment parameters in patients with and without COVID-19

\begin{tabular}{|c|c|c|c|}
\hline & COVID-19-positive & COVID-19-negative & p-value \\
\hline Patients & 313 & 195 & \\
\hline \multicolumn{4}{|l|}{ Demographics } \\
\hline Age years & $54.9 \pm 17.0$ & $58.8 \pm 18.5$ & 0.02 \\
\hline Male & $162(51.8)$ & $89(45.6)$ & 0.18 \\
\hline Race & & & $<0.01$ \\
\hline Caucasian & $11(35.5)$ & $119(61.0)$ & \\
\hline Hispanic & $160(51.1)$ & $55(28.2)$ & \\
\hline African American & $17(5.4)$ & $18(9.2)$ & \\
\hline Native American/Pacific Islander/Asian American & $25(8.0)$ & $3(1.5)$ & \\
\hline State (Arizona) & $162(51.8)$ & $119(61.0)$ & 0.04 \\
\hline Body mass index $\mathrm{kg} \cdot \mathrm{m}^{-2}$ & $33.5 \pm 12.3$ & $30.7 \pm 8.0$ & 0.04 \\
\hline Any tobacco use & $101(32.3)$ & $104(53.3)$ & $<0.01$ \\
\hline Diabetes & $97(30.9)$ & $41(21.0)$ & 0.01 \\
\hline Hyperlipidaemia & $72(23.0)$ & $38(19.5)$ & 0.35 \\
\hline Hypertension & $125(39.9)$ & $82(42.1)$ & 0.64 \\
\hline Heart failure & $12(3.83)$ & $23(11.8)$ & $<0.01$ \\
\hline Chronic kidney disease & $10(3.2)$ & $6(3.1)$ & 0.94 \\
\hline Asthma & $32(10.2)$ & $25(12.8)$ & 0.37 \\
\hline COPD/emphysema & $15(4.8)$ & $28(14.4)$ & $<0.01$ \\
\hline Cerebral vascular disease & $12(3.8)$ & 7 (3.6) & 0.88 \\
\hline Transplant & $0(0.0)$ & $2(1.0)$ & 0.15 \\
\hline Cancer & $13(4.2)$ & $18(9.2)$ & 0.02 \\
\hline Autoimmune disease & $8(2.6)$ & $11(5.6)$ & 0.08 \\
\hline Coronary artery disease & $20(6.4)$ & $24(12.3)$ & 0.02 \\
\hline Hepatitis C & $4(1.3)$ & $9(4.6)$ & 0.02 \\
\hline Pulmonary hypertension & $2(0.6)$ & $5(2.6)$ & 0.11 \\
\hline Cirrhosis & $0(0.0)$ & $2(1.0)$ & 0.15 \\
\hline \multicolumn{4}{|l|}{ Laboratory values } \\
\hline White blood cell count $\times 10^{3} \mathrm{~mm}^{-3}$ & $8.8 \pm 15.1$ & $13.2 \pm 25.9$ & $<0.01$ \\
\hline Lymphocyte count $\times 10^{3} \mu \mathrm{L}^{-1}$ & $2.4 \pm 14.5$ & $1.7 \pm 1.2$ & $<0.01$ \\
\hline Haemoglobin $\mathrm{g} \cdot \mathrm{dL}^{-1}$ & $13.8 \pm 1.9$ & $12.9 \pm 2.4$ & $<0.01$ \\
\hline Haematocrit \% & $41.5 \pm 5.2$ & $39.7 \pm 6.7$ & $<0.01$ \\
\hline Platelet count $\times 10^{3} \mathrm{~mm}^{-3}$ & $220.4 \pm 87.5$ & $262 \pm 105.0$ & $<0.01$ \\
\hline Creatinine $\mathrm{mg} \cdot \mathrm{dL}^{-1}$ & $1.13 \pm 0.9$ & $1.27 \pm 1.7$ & 0.26 \\
\hline eGFR $\mathrm{mL} \cdot \mathrm{min}^{-1} \cdot 1.63 \mathrm{~m}^{-2}$ & $78.5 \pm 29.8$ & $77.1 \pm 30.7$ & 0.65 \\
\hline NT-proBNP pg.mL ${ }^{-1}$ & $1247.2 \pm 3663.3$ & $5582.7 \pm 15837.4$ & $<0.01$ \\
\hline \multicolumn{4}{|l|}{ Medical therapy } \\
\hline Anticoagulation & & & $<0.01$ \\
\hline None & $49(15.7)$ & $62(31.8)$ & \\
\hline Full dose & $49(15.7)$ & $30(15.4)$ & \\
\hline Prophylaxis & $215(68.7)$ & $103(52.8)$ & \\
\hline Steroids & $171(54.6)$ & $59(30.3)$ & $<0.01$ \\
\hline Azithromycin & $252(80.5)$ & $99(50.8)$ & $<0.01$ \\
\hline Remdesivir & $23(7.4)$ & $1(0.5)$ & $<0.01$ \\
\hline
\end{tabular}

were from Arizona ( $\mathrm{p}=0.04)$. Compared with COVID-19-negative patients, COVID-19-positive patients were younger ( $54.9 \pm 17.0$ versus $58.8 \pm 18.5$ years; $\mathrm{p}=0.02)$, from a racial/ethnic minority $(\mathrm{p}<0.01)$, had higher body mass index $\left(33.5 \pm 12.3\right.$ versus $\left.30.7 \pm 8.0 \mathrm{~kg} \cdot \mathrm{m}^{-2} ; \mathrm{p}<0.01\right)$ and more likely to be diabetic (30.9\% versus 21.0\%; $\mathrm{p}=0.01$ ). COVID-19-positive patients had a lower incidence of tobacco use (32.3\% versus 53.3\%; $\mathrm{p}<0.01$ ), COPD/emphysema ( $4.8 \%$ versus $14.4 \% ; \mathrm{p}<0.01)$, heart failure ( $3.8 \%$ versus $11.8 \%$; $\mathrm{p}<0.01)$, coronary artery disease $(6.4 \%$ versus $12.3 \%$; $=0.02)$ and cancer $(4.2 \%$ versus $9.2 \%$; $\mathrm{p}=0.02)$. Additional demographic data are reported in table 1 .

Radiology reports from chest CT scans stated findings of COVID-19 or atypical/viral pneumonia in $91.1 \%$ of COVID-19-positive patients and 32.3\% of COVID-19-negative patients $(\mathrm{p}<0.01)$ (table 2). A normal CT scan was reported in 3.2\% of COVID-19-positive patients and 24.6\% of COVID-19-negative patients 
TABLE 2 Computed tomography (CT) findings in patients with and without COVID-19

\begin{tabular}{|c|c|c|c|}
\hline & COVID-19-positive & COVID-19-negative & $\mathrm{p}$-value \\
\hline Patients & 313 & 195 & \\
\hline \multicolumn{4}{|l|}{ Type of CT scan } \\
\hline Contrast enhanced CT & $197(62.9)$ & $147(75.3)$ & $<0.01$ \\
\hline \multicolumn{4}{|l|}{ Radiologist interpretation } \\
\hline COVID-19/atypical pneumonia & $285(91.1)$ & $63(32.3)$ & $<0.01$ \\
\hline Pulmonary embolism & $8(4.2)$ & $9(6.3)$ & 0.45 \\
\hline Normal & $10(3.2)$ & $48(24.6)$ & $<0.01$ \\
\hline Non-COVID-19 pneumonia & $9(2.9)$ & $27(13.9)$ & $<0.01$ \\
\hline Pulmonary oedema & $4(1.3)$ & $10(5.1)$ & 0.01 \\
\hline Emphysema & $2(0.6)$ & $9(4.6)$ & $<0.01$ \\
\hline Tumour & $1(0.3)$ & $11(5.6)$ & $<0.01$ \\
\hline Aspiration/bronchitis & $1(0.3)$ & $9(4.6)$ & $<0.01$ \\
\hline Pleural effusion & $1(0.3)$ & $13(6.7)$ & $<0.01$ \\
\hline Pulmonary infarct & $0(0.0)$ & $4(2.1)$ & 0.02 \\
\hline Interstitial lung disease & $0(0.0)$ & $1(0.5)$ & 0.38 \\
\hline \multicolumn{4}{|l|}{ Visual CT analysis } \\
\hline CT severity score & $9.7 \pm 5.4$ & $6.9 \pm 5.3^{\#}$ & $<0.01$ \\
\hline \multicolumn{4}{|l|}{ Quantitative $\mathrm{CT}$ analysis } \\
\hline BV $5 \%$ & $25.3 \pm 7.4$ & $30.1 \pm 9.6$ & $<0.01$ \\
\hline BV5-10\% & $22.6 \pm 3.7$ & $25.0 \pm 3.5$ & $<0.01$ \\
\hline BV10\% & $52.0 \pm 9.7$ & $45.2 \pm 10.9$ & $<0.01$ \\
\hline Total blood volume $\mathrm{mL}$ & $210 \pm 72$ & $215 \pm 72$ & 0.45 \\
\hline Total lung volume $\mathrm{mL}$ & $3034 \pm 1203$ & $2839 \pm 1123$ & 0.06 \\
\hline Peak area $\mathrm{mm}^{2}$ & $9.4 \pm 3.1$ & $11.4 \pm 3.8$ & $<0.01$ \\
\hline Peak volume $\%$ total blood volume & $24.4 \pm 3.7$ & $24.1 \pm 3.4$ & 0.40 \\
\hline AUC & $272.8 \pm 32.9$ & $264.7 \pm 35.7$ & 0.02 \\
\hline Weight mean $\mathrm{mm}^{2}$ & $17.9 \pm 4.0$ & $19.4 \pm 4.3$ & $<0.01$ \\
\hline Kurtosis & $-0.7 \pm 0.4$ & $-0.8 \pm 0.3$ & 0.64 \\
\hline Skewness & $0.8 \pm 0.2$ & $0.8 \pm 0.2$ & 0.56 \\
\hline \multicolumn{4}{|c|}{$\begin{array}{l}\text { Data are presented as } \mathrm{n}, \text { mean } \pm \text { SD or } \mathrm{n}(\%) \text {, unless otherwise stated. BV } 5 \%, \mathrm{BV} 5-10 \% \text { and } \mathrm{BV} 10 \% \text { : percentage of } \\
\text { blood volume in vessels with a cross-sectional area between } 1.25 \text { and } 5 \mathrm{~mm}^{2} \text {, between } 5-10 \mathrm{~mm}^{2} \text { and }>10 \mathrm{~mm}^{2} \text {, } \\
\text { respectively, relative to the total pulmonary blood volume; AUC: area under the receiver operating characteristic } \\
\text { curve. "\#: limited to the } 90 \text { patients with findings of COVID-19/atypical pneumonia or non-COVID-19 pneumonia } \\
\text { on CT. }\end{array}$} \\
\hline
\end{tabular}

$(\mathrm{p}<0.01)$. There was no significant difference in the frequency of pulmonary embolism $(\mathrm{p}=0.45)$. Patients with COVID-19 were significantly less likely to have findings of non-COVID-19 pneumonia (2.9\% versus 13.9\%; $\mathrm{p}<0.01$ ), pulmonary oedema ( $1.3 \%$ versus $5.1 \%$; $\mathrm{p}=0.01)$, emphysema $(0.6 \%$ versus $4.6 \%$; $\mathrm{p}<0.01)$, tumour $(0.3 \%$ versus $5.6 \% ; \mathrm{p}<0.01)$, aspiration/bronchitis $(0.3 \%$ versus $4.6 \%$; $<0.01)$, pleural effusion $(0.3 \%$ versus $6.7 \%)$ or pulmonary infarct $(0 \%$ versus $2.1 \%$; $\mathrm{p}=0.02)$.

On quantitative CT analysis (table 2), patients with COVID-19 had significantly lower BV5\% compared with COVID-19-negative patients (25.3 $\pm 7.4 \%$ versus $30.1 \pm 9.6 \%$; $\mathrm{p}<0.01)$. BV5\% was also significantly lower for the subset of patients with COVID-19 and CT findings of COVID-19/atypical pneumonia (23.7 $\pm 7.3 \%$ versus 27.2 $\pm 7.2 \%$; $\mathrm{p}=0.02$ ). Patients with COVID-19 had a significantly higher severity score compared with

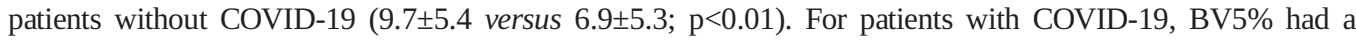
moderate correlation with the severity score (Spearman's $\rho=-0.45, \mathrm{p}<0.0001$ ) (figure 3 ). Differences in BV5\% and Spearman's $\rho$ stratified by CT findings are reported in supplementary table S1. There were no significant differences in total lung volume (3034 \pm 1203 versus $2839 \pm 1123 \mathrm{~mL}$; $\mathrm{p}=0.06)$ and total blood volume (210 \pm 72 versus $215 \pm 72 \mathrm{~mL}$; $\mathrm{p}=0.45$ ) in COVID-19-positive versus COVID-19-negative patients, respectively. CT scans from COVID-19-positive patients had a lower peak area (9.4 \pm 3.1 versus $\left.11.4 \pm 3.8 \mathrm{~mm}^{2} ; \mathrm{p}<0.01\right)$ and weight mean $\left(17.9 \pm 4.0\right.$ versus $\left.19.4 \pm 4.3 \mathrm{~mm}^{2} ; \mathrm{p}<0.01\right)$, and higher area under the curve (272.8 \pm 32.9 versus $264.7 \pm 35.7 ; \mathrm{p}=0.02)$.

There was no significant difference in BV5\% obtained from contrast enhanced CT versus noncontrast CT $(\mathrm{p}=0.23)$. Average processing time for BV5\% was $9 \mathrm{~min}$ and $22 \mathrm{~s} \mathrm{(} \pm 6 \mathrm{~min}$ and $3 \mathrm{~s}$ ), with processing time dependent on scan quality. No CT scans were unable to be analysed. 


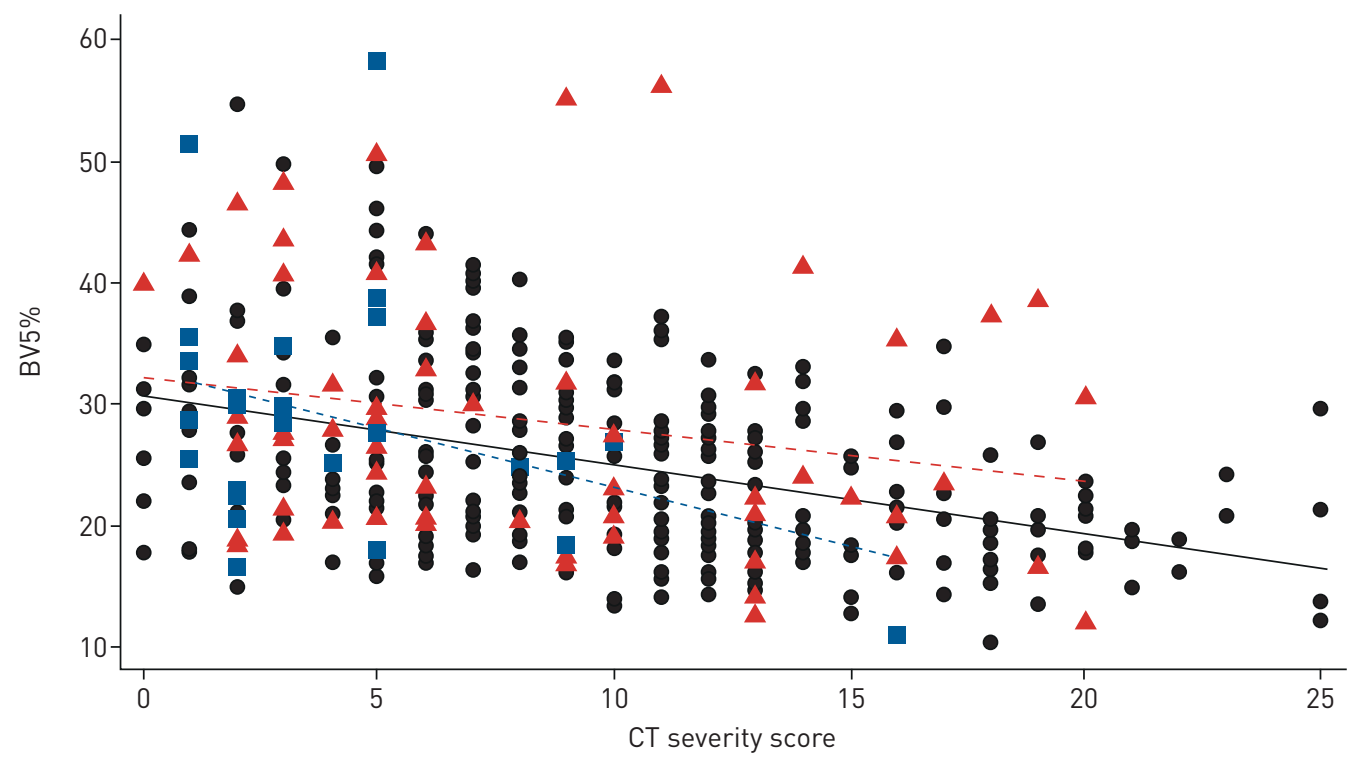

- COVID-19-positive $(\rho=-0.45, p<0.0001)$

$\Delta$ COVID-19-negative with atypical/viral pneumonia $(\rho=-0.25, p=0.047)$

- COVID-19-negative with non-COVID-19 pneumonia ( $\rho=-0.25, p=0.19$ )

FIGURE 3 Spearman's rank correlation between percentage of blood volume in vessels with a cross-sectional area between 1.25 and $5 \mathrm{~mm}^{2}$ relative to the total pulmonary blood volume (BV5\%) and visually assessed computed tomography (CT) severity score, stratified by COVID-19 status and CT findings.

Overall clinical outcomes for patients with COVID-19 were worse than for patients without COVID-19 (table 3). COVID-19 patients were hospitalised for a longer duration (8.5 \pm 10.8 versus $4.1 \pm 5.7$ days; $\mathrm{p}<0.01)$, more likely to be intubated $(20.8 \%$ versus $6.2 \%$; $<0.01)$ and trended towards higher in-hospital mortality (11.8\% versus $7.2 \%$; $\mathrm{p}=0.09)$.

In a multivariate regression analysis that did not include control for lung opacification, BV5\% remained significantly associated with mortality and intubation in patients with COVID-19 (table 4). Specifically, BV5\% as a continuous variable had OR 0.87 (95\% CI 0.79-0.96; $\mathrm{p}<0.01)$ for mortality, OR 0.89 (95\% CI 0.84-0.95; $\mathrm{p}<0.01$ ) for intubation and OR 0.90 (95\% CI 0.84-0.96; $\mathrm{p}<0.01$ ) for the composite of mortality or intubation. A BV5\% threshold of 25\% had OR 5.58 (95\% CI 1.54-20.1; $<<0.01$ ) for mortality, OR 3.20 (95\% CI 1.55-6.63; $\mathrm{p}<0.01$ ) for intubation and OR 2.54 (95\% CI 1.15-5.60; $\mathrm{p}=0.02$ ) for the composite of mortality or intubation. In patients without COVID-19 there was no significant association between BV5\% and intubation or mortality.

BV5\% was not predictive of length of hospitalisation or duration of intubation in patients with COVID-19. Length of stay was associated with BV5\% for COVID-19-negative patients $(\exp (\beta)$ 0.97, 95\% CI 0.960.98; $\mathrm{p}<0.01)$.

\section{TABLE 3 Outcomes in patients with and without COVID-19}

\begin{tabular}{lccc} 
& COVID-19-positive & COVID-19-negative & p-value \\
\hline Patients & 313 & 195 & \\
Mortality & $37(11.8)$ & $14(7.2)$ & 0.09 \\
Intubation & $65(20.8)$ & $12(6.2)$ & $<0.01$ \\
Intubation or mortality & $78(24.9)$ & $22(11.3)$ & $<0.01$ \\
Time from admission to intubation days & $2.5 \pm 3.9$ & $0.4 \pm 0.9$ & $<0.01$ \\
\hline Time intubated days & $10.7 \pm 10.8$ & $3.8 \pm 4.6$ & $<0.01$ \\
Length of stay days & $8.5 \pm 10.8$ & $4.1 \pm 5.7$ & $<0.01$ \\
\hline Length of stay $>1$ day & $267(85.3)$ & $146(74.9)$ & $<0.01$ \\
\hline Data are presented as $n$, mean \pm SD or $\mathrm{n}(\%)$, unless otherwise stated. & & \\
\hline
\end{tabular}


TABLE 4 Multivariate regression analysis of outcomes relative to percentage of blood volume in vessels with a cross-sectional area between 1.25 and $5 \mathrm{~mm}^{2}$ relative to the total pulmonary blood volume (BV5\%) as a continuous and categorical variable in patients with and without COVID-19

\begin{tabular}{|c|c|c|c|c|}
\hline & \multicolumn{2}{|c|}{ COVID-19-positive } & \multicolumn{2}{|c|}{ COVID-19-negative } \\
\hline & Association $^{\#}$ & p-value & Association $^{\#}$ & $p$-value \\
\hline \multicolumn{5}{|l|}{ Mortality } \\
\hline BV5\% continuous & $0.87(0.79-0.96)$ & $<0.01$ & $1.02(0.94-1.10)$ & 0.61 \\
\hline BV5\% categorical $<25 \%$ & $5.58(1.54-20.1)$ & $<0.01$ & $0.50(0.08-3.24)$ & 0.46 \\
\hline \multicolumn{5}{|l|}{ Intubation } \\
\hline BV5\% continuous & $0.89(0.84-0.95)$ & $<0.01$ & $1.00(0.91-1.10)$ & 0.96 \\
\hline BV5\% categorical $<25 \%$ & $3.20(1.55-6.63)$ & $<0.01$ & $3.31(0.59-18.5)$ & 0.17 \\
\hline \multicolumn{5}{|l|}{ Mortality or intubation } \\
\hline BV5\% continuous & $0.90(0.84-0.96)$ & $<0.01$ & $0.99(0.93-1.05)$ & 0.69 \\
\hline BV5\% categorical $<25 \%$ & $2.54(1.15-5.60)$ & $<0.01$ & $2.05(0.62-6.73)$ & 0.24 \\
\hline \multicolumn{5}{|l|}{ Length of stay } \\
\hline BV5\% continuous & $0.98(0.96-1.00)$ & 0.08 & $0.97(0.96-0.98)$ & $<0.01$ \\
\hline \multicolumn{5}{|l|}{ Time intubated } \\
\hline BV5\% continuous & $0.99(0.93-1.06)$ & 0.83 & $1.02(0.95-1.09)$ & 0.51 \\
\hline
\end{tabular}

After adding the visually assessed CT severity score to the multivariate regression analysis, a BV5\% threshold of $25 \%$ remained significantly associated with mortality in patients with COVID-19, with OR 4.27 (95\% CI 1.02-17.80; $\mathrm{p}=0.046$ ) for mortality. In the subset of patients without COVID-19 but with CT findings of pneumonia, BV5\% as a continuous variable had an OR 1.24 (95\% CI 1.01-1.52; p=0.039) for the composite of mortality or intubation.

For COVID-19-positive patients, a logistic regression model using age and BV5\% were chosen as features for the classifier because, after excluding treatments, they had the highest coefficients in the aforementioned multivariable linear regression for the primary outcomes. A model using age and severity score was similarly created. Age and BV5\% predicted the composite of mortality or intubation with an accuracy of $0.83 \pm 0.02$ in iterative cross-validation. Age and severity score predicted the composite of mortality or intubation with an accuracy of $0.82 \pm 0.02$ in iterative cross-validation. After training on $80 \%$ of the patients, the AUC 0.85 for age and BV5\% and the AUC 0.87 for age and severity score were not significantly different $(\mathrm{p}=0.18)$ (figure $4 \mathrm{a})$. The model was also used to forecast risk for the composite of mortality or intubation using simulated data of patients between 50 and 80 years old and a BV5\% ranging from $10 \%$ to $60 \%$ (figure $4 \mathrm{~b}$ ).

\section{Discussion}

In this study of 508 patients presenting to the hospital, tested for COVID-19 and undergoing chest CT within $24 \mathrm{~h}$ of presentation, BV5\% from COVID-19 patients was significantly lower than BV5\% from a heterogenous cohort of patients without COVID-19. This difference was driven mainly by patients with CT findings of COVID-19/atypical pneumonia. BV5\% was predictive of outcomes in COVID-19 patients in a multivariate model that did not account for lung opacification, with a BV5\% threshold below 25\% associated with OR 5.58 for mortality, OR 3.20 for intubation and OR 2.54 for the composite of mortality or intubation. After including the severity of lung opacification in the multivariate analysis, a BV5\% threshold of 25\% remained significantly associated with mortality, with OR 4.27.

In healthy patients, BV5\% constitutes the majority of pulmonary blood volume distribution [13] and is closely correlated with histological assessment of vascular cross-sectional area [14]. Alterations in BV5\% are not exclusive to COVID-19 and can manifest in other diseases that diffusely affect pulmonary perfusion, e.g. COPD [15] and acute respiratory distress syndrome [7]. In these cohorts, chronic vascular remodelling and small vessel loss are the underlying aetiology for reduced BV5\% [16]. The reduction in BV5\% in COVID-19 may reflect the acute sequela of microcirculatory disruption induced by SARS-CoV-2 [5]; however, the extent to which other processes such as ventilation/perfusion mismatch and shunting impact BV5\% are unknown. The significant association between reduced BV5\% and mortality in COVID-19 suggests that BV5\% may offer insights into the underlying pathological processes involved in 

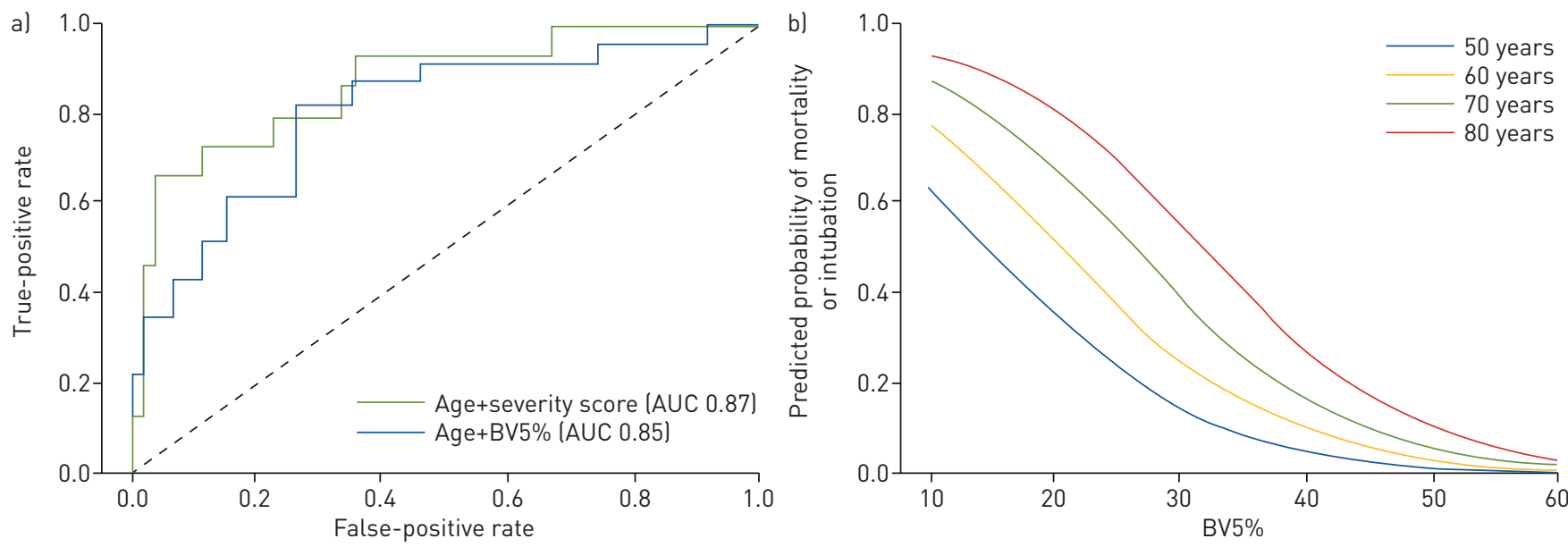

FIGURE 4 a) Receiver operating characteristic (ROC) curve from the testing set of the logistic regression model. Patient age and percentage of blood volume in vessels with a cross-sectional area between 1.25 and $5 \mathrm{~mm}^{2}$ relative to the total pulmonary blood volume (BV5\%) have an area under the ROC curve (AUC) 0.85 to predict the composite of mortality or intubation in patients with COVID-19, not significantly different from AUC 0.87 for age and visually assessed computed tomography severity score $(p=0.18)$. b) Probability of the composite of mortality or intubation for simulated COVID-19 patients aged $50,60,70$ and 80 years with BV5\% ranging from $10 \%$ to $60 \%$.

SARS-CoV-2 infection and potentially serve as a tool for quantifying the extent of these processes in an acute care setting.

Models using BV5\% and severity score had high AUCs for the composite of mortality or intubation in COVID-19 patients, supporting their role as imaging surrogates for disease severity. However, BV5\% and severity score are only moderately correlated, and the presence of a low BV5\% in patients with a low severity score may signify that changes in pulmonary vascular volume distribution represent either an early indicator of disease severity or a distinct (if low-frequency) phenotype. Since the severity score is a general measure of lung opacification in COVID-19, it can be influenced by alveolar disease (e.g. diffuse alveolar damage), interstitial changes (e.g. fibrosis), vascular impairment (e.g. haemorrhage) or a combination of these processes [17-19], thus posing challenges in using the severity score to identify targeted treatments. However, because $\mathrm{BV} 5 \%$ is closely correlated with histological assessment of vascular cross-sectional area [14], we speculate that BV5\% may be able to inform possible treatment pathways for COVID-19, e.g. by identifying patients that would benefit from anticoagulation.

Based on apilot study of 97 patients (not published), the association between BV5\% and outcomes in COVID-19 patients was significant only for CT scans performed within $24 \mathrm{~h}$ of presentation. Beyond this window an increasing number of variables likely obscure the prognostic impact of BV5\%, especially given the absence of clear guidelines regarding hospitalisation at the time the study data were collected during the initial phase of the pandemic. This may also explain why BV5\% was not predictive of duration of hospitalisation or duration of intubation in COVID-19 patients. Paradoxically, BV5\% was associated with length of stay in patients without COVID-19. In addition, after including the severity of lung opacification in the multivariate analysis, BV5\% was associated with the composite of mortality and intubation. These are subjects of current investigation.

There are several important limitations of this study. This was a retrospective study in which only a subset of patients with suspected COVID-19 underwent chest CT with the requisite $\leqslant 2 \mathrm{~mm}$ slice thickness. However, the results should be generalisable due to the demographically diverse population. Only one fellowship-trained cardiothoracic radiologist performed the semiquantitative visual severity scoring of CT scans and it is unknown if scoring from radiologists with different levels of expertise would have the same prediction for clinical outcomes. Although only patients presenting to a hospital for acute medical care were included, these are the patients at greatest risk for poor outcome with COVID-19 [20]. It is possible that some patients with COVID-19 were miscategorised due to false-negative PCR results [21]; however, we attempted to minimise this effect by checking for additional COVID-19 PCR testing within 14 days of the initial encounter. In addition, obtaining BV5\% requires transporting the patient to a CT scanner. While limiting the movement of patients with COVID-19 throughout the hospital can mitigate exposure of the disease to healthcare personnel, the potential predictive benefit of BV5\% may outweigh this risk. 
As additional waves of the SARS-CoV-2 pandemic are expected, there is urgent need for improved diagnostic, prognostic and treatment tools. Although certain risk factors [22-24] and imaging findings [9] are associated with worse prognosis in COVID-19, accurately quantifying a patient's individual risk for progression to respiratory failure or death remains challenging. BV5\% can be quickly derived from CT scans in patients with COVID-19 seeking acute medical care, without the need for contrast material. We hypothesise that BV5\% is a unique imaging biomarker, in that it can potentially be used as a gatekeeper for identifying patients that may benefit for earlier or more aggressive therapy with anticoagulation, and we hope this study serves as a catalyst for prospective evaluation of this hypothesis.

Acknowledgements: The authors wish to thank Heather Pryzbyl and Corry Glade (Banner Health, Phoenix, AZ, USA) for assisting with the custom coding used for data retrieval. We thank Kathryn Olson (Banner - University Medical Center Phoenix, Phoenix, AZ, USA) for feedback on the manuscript.

Author contributions: M.F. Morris: conception/design; acquisition, analysis and interpretation of data; drafting the work. Y. Pershad and P. Kang: analysis and interpretation of data; drafting the work. L. Ridenour: acquisition and analysis of data; revising the draft critically for important intellectual content. B. Lavon, M. Lanclus and R. Godon: analysis and interpretation of data; revising the draft critically for important intellectual content. J. De Backer: conception/design; analysis and interpretation of data; revising the draft critically for important intellectual content. M.K. Glassberg: conception/design; acquisition, analysis and interpretation of data; revising the draft critically for important intellectual content.

Conflict of interest: M.F. Morris has nothing to disclose. Y. Pershad has nothing to disclose. P. Kang has nothing to disclose. L. Ridenour has nothing to disclose. B. Lavon is an employee of FLUIDDA, a company that develops and markets part of the technology described in this paper. M. Lanclus is an employee of FLUIDDA, a company that develops and markets part of the technology described in this paper. R. Godon is an employee of FLUIDDA, a company that develops and markets part of the technology described in this paper. J. De Backer is an employee of FLUIDDA, a company that develops and markets part of the technology described in this paper. M.K. Glassberg serves on advisory boards for Bellerophon Therapeutics, Genentech, Boehringer Ingelheim and Bristol Myers Squibb.

\section{References}

1 Cavallo JJ, Donoho DA, Forman HP. Hospital capacity and operations in the coronavirus disease 2019 (COVID-19) pandemic - planning for the Nth patient. JAMA Health Forum 2020; 1: e200345.

2 Richardson S, Hirsch JS, Narasimhan M, et al. Presenting characteristics, comorbidities, and outcomes among 5700 patients hospitalised with COVID-19 in the New York City Area. JAMA 2020; 323: 2052-2059.

3 Vahidy FS, Drews AL, Masud FN, et al. Characteristics and outcomes of COVID-19 patients during initial peak and resurgence in the Houston metropolitan area. JAMA 2020; 324: 998-1000.

4 Klok FA, Kruip MJHA, van der Meer NJM, et al. Incidence of thrombotic complications in critically ill ICU patients with COVID-19. Thromb Res 2020; 191: 148-150.

5 Ackermann M, Verleden SE, Kuehnel M, et al. Pulmonary vascular endothelialitis, thrombosis, and angiogenesis in Covid-19. N Engl J Med 2020; 383: 120-128.

6 Lang M, Som A, Carey D, et al. Pulmonary vascular manifestations of COVID-19 pneumonia. Radiol Cardiothorac Imaging 2020; 2: e200277.

7 Thillai M, Patvardhan C, Swietlik EM, et al. Functional respiratory imaging identifies redistribution of pulmonary blood flow in patients with COVID-19. Thorax 2021; 76: 182-184.

8 Pan F, Ye T, Sun P, et al. Time course of lung changes at chest CT during recovery. Radiology 2020; 295: 715-721.

9 Francone M, lafrate F, Masci GM, et al. Chest CT score in COVID-19 patients: correlation with disease severity and short-term prognosis. Eur Radiol 2020; 30: 6808-6817.

10 Lins M, Vandevenne J, Thillai M, et al. Assessment of small pulmonary blood vessels in COVID-19 patients using HRCT. Acad Radiol 2020; 27: 1449-1455.

11 Pedregosa F, Michel V, Grisel O, et al. Scikit-learn: machine learning in Python. J Mach Learn Res 2011; 12: 2825-2830.

12 Pershad Y, Guo M, Altman RB. Pathway and network embedding methods for prioritising psychiatric drugs. Pac Symp Biocomput 2020; 25: 671-682.

13 Estépar RSJ, Kinney GL, Black-Shinn JL, et al. Computed tomographic measures of pulmonary vascular morphology in smokers and their clinical implications. Am J Respir Crit Care Med 2013; 188: 231-239.

14 Rahaghi FN, Argemí G, Nardelli P, et al. Pulmonary vascular density: comparison of findings on computed tomography imaging with histology. Eur Respir J 2019; 54: 1900370.

15 Washko GR, Nardelli P, Ash SY, et al. Arterial vascular pruning, right ventricular size, and clinical outcomes in chronic obstructive pulmonary disease. A longitudinal observational study. Am J Respir Crit Care Med 2019; 200: 454-461. 
16 Synn AJ, Li W, San José Estépar R, et al. Pulmonary vascular pruning on computed tomography and risk of death in the Framingham Heart Study. Am J Respir Crit Care Med 2021; 203: 251-254.

17 Peys E, Stevens D, Weygaerde YV, et al. Haemoptysis as the first presentation of COVID-19: a case report. BMC Pulm Med 2020; 20: 275.

18 Polak SB, Van Gool IC, Cohen D, et al. A systematic review of pathological findings in COVID-19: a pathophysiological timeline and possible mechanisms of disease progression. Mod Pathol 2020; 33: 2128-2138.

19 Zhang $\mathrm{H}$, Zhou $\mathrm{P}$, Wei $\mathrm{Y}$, et al. Histopathologic changes and SARS-CoV-2 immunostaining in the lung of a patient with COVID-19. Ann Intern Med 2020; 172: 629-632.

20 Karagiannidis C, Mostert C, Hentschker C, et al. Case characteristics, resource use, and outcomes of 10021 patients with COVID-19 admitted to 920 German hospitals: an observational study. Lancet Respir Med 2020; 8: 853-862.

21 Wiersinga WJ, Rhodes A, Cheng AC, et al. Pathophysiology, transmission, diagnosis, and treatment of coronavirus disease 2019 (COVID-19). JAMA 2020; 324: 782.

22 Williamson EJ, Walker AJ, Bhaskaran K, et al. Factors associated with COVID-19-related death using OpenSAFELY. Nature 2020; 584: 430-436.

23 Homayounieh F, Ebrahimian S, Babaei R, et al. CT radiomics, radiologists and clinical information in predicting outcome of patients with COVID-19 pneumonia. Radiol Cardiothorac Imaging 2020; 2: e200322.

24 Danwang C, Endomba FT, Nkeck JR, et al. A meta-analysis of potential biomarkers associated with severity of coronavirus disease 2019 (COVID-19). Biomark Res 2020; 8: 37. 